\title{
Bullae and Hyperpigmented Patches on the Legs
}

Paul A. Regan, MD; Bryan E. Anderson, MD; Matthew F. Helm, MD

Eligible for 1 MOC SA Credit From the ABD

This Photo Challenge in our print edition is eligible for 1 self-assessment credit for Maintenance of Certification from the American Board of Dermatology (ABD). After completing this activity, diplomates can visit the ABD website (http://www.abderm.org) to self-report the credits under the activity title "Cutis Photo Challenge." You may report the credit after each activity is completed or after accumulating multiple credits.

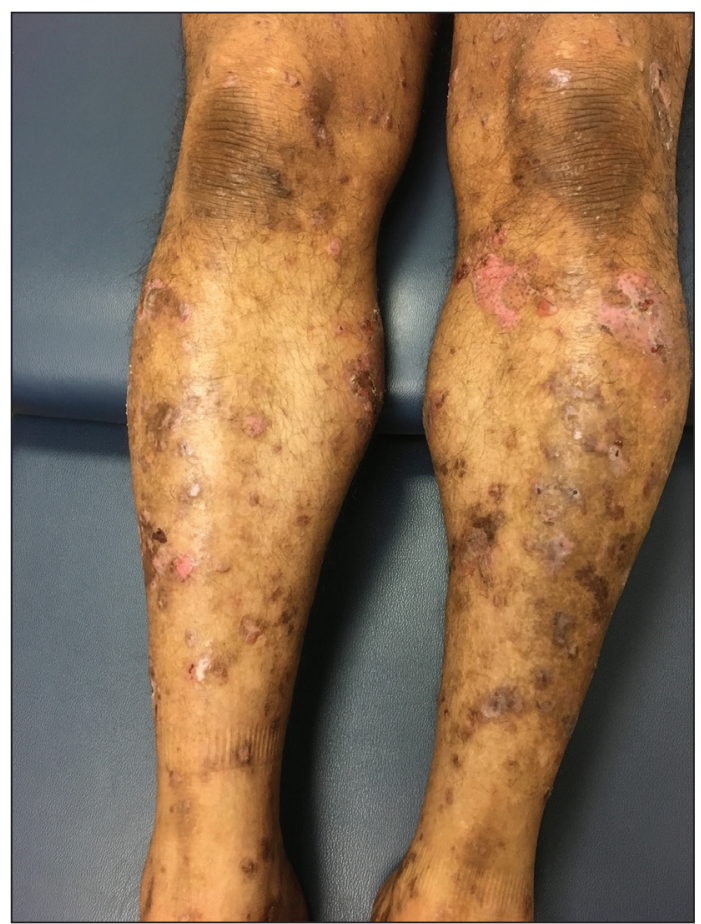

A 50-year-old man presented with a pruritic bullous dermatosis on the lower legs, arms, and back of 1 month's duration. He had an 8-year history of lichen planus, and the lesions recently had worsened despite the addition of UVB phototherapy. His medical history was remarkable for hepatitis B treated with entecavir and the addition of hydrochlorothiazide for essential hypertension 2 weeks prior to the dramatic worsening of the rash. Physical examination revealed multiple bullae on the lower legs associated with violaceous and hyperpigmented papules and patches. He also had violaceous papules on the lower back and eroded lesions on the oral mucosa. Shave biopsies were obtained from the right thigh and mid back, and histopathologic analysis was performed for both routine histology and direct immunofluorescence.

\section{WHAT'S YOUR DIAGNOSIS?}
a. bullous lichen planus
b. bullous lupus erythematosus
c. bullous pemphigoid
d. lichen planus actinicus
e. lichen planus pemphigoides

\footnotetext{
From the Department of Dermatology, Penn State Health Milton S. Hershey Medical Center, Pennsylvania.

The authors report no conflict of interest.

Correspondence: Paul A. Regan, MD, Penn State Health Milton S. Hershey Medical Center, 500 University Dr, HU14, Hershey, PA 17033 (pregan@pennstatehealth.psu.edu).

doi:10.12788/cutis.0079
} 


\section{THE DIAGNOSIS: Lichen Planus Pemphigoides}

$\Lambda$ skin biopsy from the right thigh demonstrated subepidermal blisters containing neutrophils (Figure 1). Direct immunofluorescence revealed linear basement membrane zone staining with $\mathrm{C} 3$ and trace staining with $\operatorname{IgG}$ (Figure 2), supporting a diagnosis of lichen planus pemphigoides (LPP). Oral prednisonestarting at $60 \mathrm{mg}$ daily and tapered to $40 \mathrm{mg}$ for a week, $20 \mathrm{mg}$ for a week, then $10 \mathrm{mg}$ for a month-along with triamcinolone ointment $0.1 \%$ to affected areas led to improvement. Hydrochlorothiazide and UV light therapy were discontinued. Doxycycline $100 \mathrm{mg}$ twice daily and nicotinamide $500 \mathrm{mg}$ twice daily prescribed as adjunctive therapy also led to improvement. The patient achieved remission with doxycycline and was doing well without prednisone; however, he experienced a flare of his disease about 6 months later and was started on mycophenolate mofetil $1 \mathrm{~g}$ twice daily after clearance from his gastroenterologist, given his history of hepatitis B. He has been doing well since starting mycophenolate mofetil.

Lichen planus pemphigoides is a rare autoimmune bullous dermatosis with features of both lichen planus and bullous pemphigoid. ${ }^{1}$ Violaceous papules and tense bullae may be superimposed or arise independently. The chest, abdomen, back, and upper and lower extremities typically are involved. ${ }^{2}$ Oral mucosal involvement with white reticular streaks or erosions and nail involvement have been reported. ${ }^{2}$ Histopathologic and immunologic findings establish the diagnosis. Lichen planus pemphigoides is associated with subepidermal bullae and linear deposits of IgG and C3 on the basement membrane zone. ${ }^{1}$ Autoantibodies to bullous pemphigoid (BP) antigens BP180 and BP230 are associated with LPP. $^{3}$

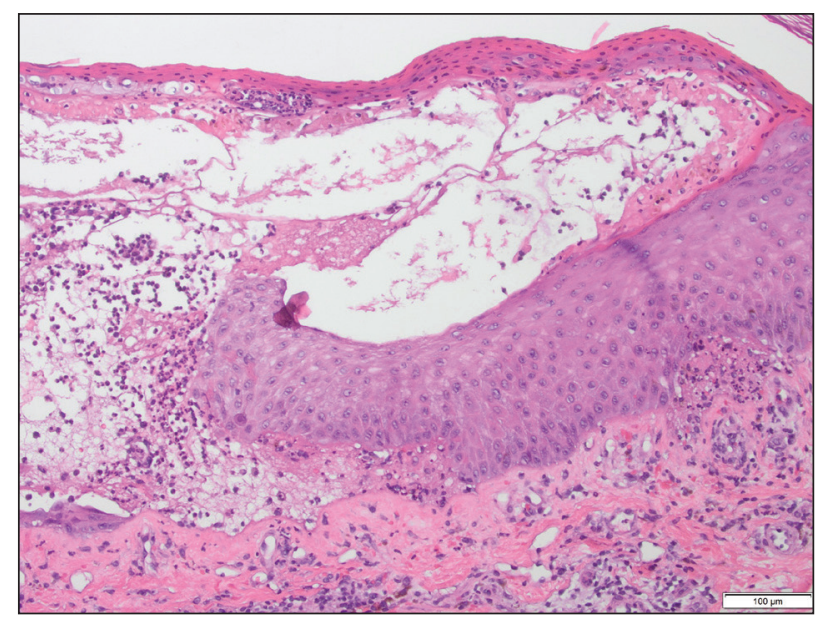

FIGURE 1. Lichen planus pemphigoides. Biopsy showed a subepidermal bulla containing neutrophils (H\&E, original magnification $\times 10)$.
The pathogenesis of LPP remains unclear, but there are associations with chronic diseases, medications, and certain therapies. ${ }^{14-6}$ Several case reports have linked LPP to chronic viral hepatitis infections, as well as malignant tumors of the skin, mucosa, and gastrointestinal tract. ${ }^{2}$ Lichen planus pemphigoides has been reported in a patient on entecavir for hepatitis B as well as in a patient treated for hepatitis $C$ with interferon and ribavirin. ${ }^{1}$ Lichen planus pemphigoides has been described in patients treated with the angiotensinconverting enzyme inhibitors enalapril, captopril, and ramipril. ${ }^{4,5,7}$ UV phototherapy also has been associated with the development of LPP. ${ }^{6}$ Hydrochlorothiazide previously has been reported as a cause of druginduced lichen planus. ${ }^{8}$ A PubMed search of articles indexed for MEDLINE using the terms lichen planus pemphigoides and hydrochlorothiazide revealed no reports of hydrochlorothiazide-induced LPP.

Lichen planus pemphigoides demonstrates overlap with other blistering dermatoses, such as BP, bullous lupus erythematosus, and bullous lichen planus. Although histologically and immunologically similar to BP, LPP can be differentiated clinically by the presence of violaceous papules or plaques typical of lichen planus. ${ }^{9}$ Bullous pemphigoid is more common in individuals older than 70 years, whereas LPP tends to occur in middle-aged adults. ${ }^{2}$ Bullous lupus erythematosus usually is associated with manifestations of systemic lupus erythematosus and autoantibodies to collagen type VII. ${ }^{10}$ Salt-split skin studies demonstrate immunofluorescence on the dermal side of the split. Individuals affected by

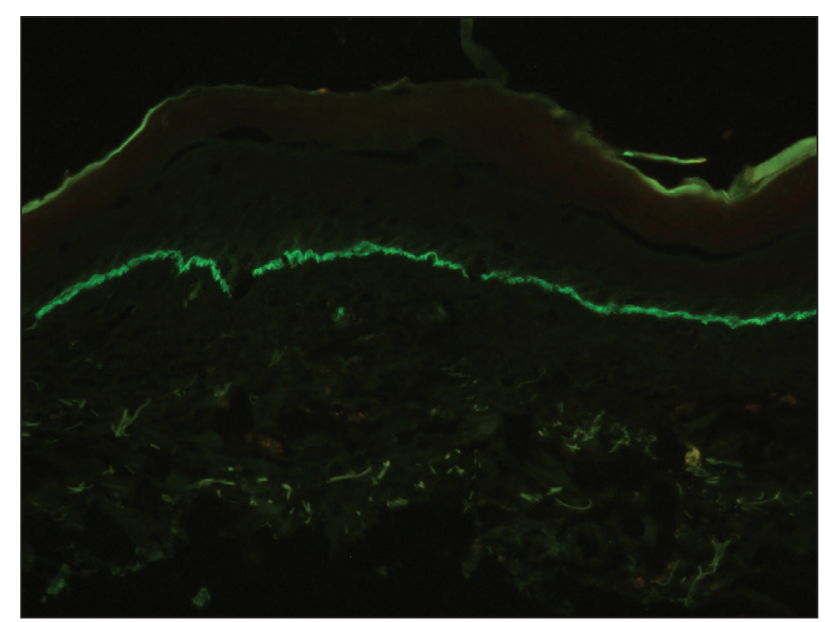

FIGURE 2. Lichen planus pemphigoides. Direct immunofluorescence revealed linear $\mathrm{C} 3$ deposition along the basement membrane zone and trace lgG (original magnification $\times 40$ ). 
bullous lupus erythematosus typically have a history of photosensitivity. ${ }^{10}$ Blisters in LPP may form de novo from unaffected skin, whereas the bullae in bullous lichen planus are limited to existing lichenoid papules. ${ }^{9}$ The autoantibodies typical of LPP are absent in bullous lichen planus. Lichen planus actinicus is a variant of lichen planus that presents with annular, dyschromic, or violaceous plaques in a photodistributed pattern without bullous lesions. ${ }^{9}$

Lichen planus pemphigoides most commonly is treated with systemic corticosteroids. Topical steroids, dapsone, erythromycin, tetracycline and nicotinamide, azathioprine, and mycophenolate mofetil have been reported as adjuncts to systemic steroid therapy., ${ }^{2,11}$ Most reports describe treatment success with resolution of blistering lesions.

\section{REFERENCES}

1. Jang SH, Yun SJ, Lee SC, et al. Lichen planus pemphigoides associated with chronic hepatitis B virus infection. Clin Exp Dermatol. 2015;40:868-871.
2. Zaraa I, Mahfoudh A, Sellami MK, et al. Lichen planus pemphigoides: four new cases and a review of the literature. Int J Dermatol. 2013;52:406-412.

3. Harting MS, Hsu S. Lichen planus pemphigoides: a case report and review of the literature. Dermatol Online J. 2006;12:10.

4. Onprasert W, Chanprapaph K. Lichen planus pemphigoides induced by enalapril: a case report and a review of literature. Case Rep Dermatol. 2017;9:217-224.

5. Ben Salem C, Chengeul L, Ghariani N, et al. Captopril-induced lichen planus pemphigoides. Pharmacoepidemiol Drug Saf. 2008;17:722-724.

6. Kuramoto N, Kishimoto S, Shibagaki R, et al. PUVA-induced lichen planus pemphigoides. Br J Dermatol. 2000;142:509-512.

7. Zhu YI, Fitzpatrick JE, Kornfield BW. Lichen planus pemphigoides associated with Ramipril. Int J Dermatol. 2006;45:1453-1455.

8. Sin B, Miller M, Chew E. Hydrochlorothiazide induced lichen planus in the emergency department. J Pharm Pract. 2017;30:266-269.

9. Weston G, Payette M. Update on lichen planus and its clinical variants. Int J Women Dermatol. 2015;1:140-149.

10. Contestable JJ, Edhegard KD, Meyerle JH. Bullous systemic lupus erythematosus: a review and update to diagnosis and treatment. Am J Clin Dermatol. 2014;15:517-524.

11. Fivenson DP, Kimbrough TL. Lichen planus pemphigoides: combination therapy with tetracycline and nicotinamide. J Am Acad Dermatol. 1997;36:638-640 